\title{
High expression of NUCB2 promotes papillary thyroid cancer cells proliferation and invasion
}

This article was published in the following Dove Medical Press journal:

OncoTargets and Therapy

\author{
Jingzhu Zhao* \\ Xinwei Yun* \\ Xianhui Ruan \\ Jiadong Chi \\ Yang Yu \\ Yigong Li \\ Xiangqian Zheng \\ Ming Gao
}

Department of Thyroid and Neck Tumor, Tianjin Medical University Cancer Institute and Hospital, National Clinical Research Center for Cancer, Key Laboratory of Cancer Prevention and Therapy, Tianjin's Clinical Research Center for Cancer, Tianjin 300060, PR China

*These authors contributed equally to this work

\footnotetext{
Correspondence: Ming Gao; Xiangqian Zheng

Department of Thyroid and Neck Tumor, Tianjin Medical University Cancer Institute and Hospital, National Clinical Research Center for Cancer, Key Laboratory of Cancer Prevention and Therapy, Tianjin's Clinical Research Center for Cancer, Huanhuxi Road, Ti-Yuan-Bei, Hexi District, Tianjin 300060, PR China

Tel $+86 I 8622162632$

Fax +8622 23355901

Email headandneck15@aliyun.com
}

\begin{abstract}
Background: NUCB2, a novel multifunctional protein containing several functional domains, was newly found to play important roles in many cancers, but its role in papillary thyroid cancer (PTC) is not well investigated. Therefore, our study was performed to explore the functions of NUCB2 in PTC.

Methods: NUCB2 protein level was analyzed by immunohistochemistry. Data analyses were made by performing chi-squared test. Quantitative reverse-transcription PCR, Western blot, colony formation, MTT, and transwell invasion assays were performed to test the expression levels and functions of NUCB2 in PTC.

Results: In PTC tissues, NUCB2 protein expression level was positively correlated with extrathyroidal extension, TNM stage, and tumor size of PTC patients. In vitro experiments demonstrated that knockdown of NUCB2 using specific shRNA for NUCB2 significantly impaired cell proliferation and invasion of PTC cell lines. In vivo, silencing of NUCB2 inhibited the growth of tumors in mice.

Conclusion: These results suggested a novel function of NUCB2 in the process of proliferation and invasion in PTC. NUCB2 may be considered a potent prognostic factor in PTC.

Keywords: NUCB2, papillary thyroid cancer, proliferation, invasion
\end{abstract}

\section{Introduction}

The incidence of thyroid cancer is gradually increasing in recent years. ${ }^{1}$ Nowadays, thyroid cancer is the fifth most common cancer in women in the USA, and over 53,990 cases have been newly diagnosed in men and women in $2018 .^{2}$ This rise may be partly due to the widespread and developmental screening. Thyroid cancer is a malignant disease exhibiting heterogenicity, with several histological types and subtypes. The preferred treatment for most patients with thyroid cancer is still surgery and thyroidstimulating hormone suppression, ${ }^{3}$ while other options include radioactive iodine therapy. Papillary thyroid cancer (PTC) is well-differentiated and usually associated with good prognosis and therapeutic response; nevertheless, about $10 \%$ of patients die from recurrences and distant metastasis after several years of diagnosis. ${ }^{4}$ Nowadays, research on cancer genomics, risk markers, and targeted therapies may be the key to resolving the problem.

NUCB2/nesfatin-1, first identified by Ohi et al in 2006, was originally found in hypothalamic nuclei and play important role in food intake and energy homeostasis. ${ }^{5}$ NUCB2 protein consists of several functional domains such as a signal peptide, a $\mathrm{Leu} / \mathrm{Ile}$ rich region, two $\mathrm{Ca}^{2+}$ binding EF-hand domains separated by an acidic amino acid rich region, and a leucine zipper, ${ }^{6,7}$ and is associated with basic cellular functions. ${ }^{5,8,9}$ Recently, several studies have indicated that NUCB2 is also expressed in 
several human peripheral tissues, including the stomach, pancreas, testis, and adipose tissues. ${ }^{8-11}$ Other related metabolic functions of NUCB2 include insulin release, adipocyte differentiation, and myocardial performance. ${ }^{12}$ Additionally, many studies have identified that NUCB2 may be associated with tumor behavior. ${ }^{13-15}$ Until recently, a study found an upregulation of NUCB2 in thyroid cancer by transcriptome analysis. ${ }^{16}$ However, there is still a shortage of studies investigating the correlation between NUCB2 and thyroid cancer.

In this study, we systematically investigated the roles of NUCB2 in PTC. We demonstrated that NUCB2 expression is significantly positively correlated with PTC progression. We further reported that knockdown of NUCB2 impaired cell proliferation and invasion. Moreover, NUCB2 ablation inhibits thyroid tumorigenesis in mice. Our results strongly support a tumor-promoting role for NUCB2 in PTC.

\section{Materials and methods Patients and samples}

A total of 155 patients with thyroid cancer who have been diagnosed by pathology were incorporated into the study which was approved by our institutional research ethics committee. Fresh samples were collected just after surgery and fixed in $10 \%$ formalin before being embedded in paraffin wax. Patients' clinical and pathological data including age, gender, extrathyroidal extension, TNM stage, tumor size, lymph metastasis, and multifocality were collected. The histopathology of each specimen was reviewed by the board-certified pathologists of our institution. This study was reviewed and approved by the Ethics Committee of Tianjin Medical University Cancer Institute and Hospital. Written informed consent for the collection of tumor tissues for the study purpose was obtained from all patients. All the experiments in this study were conducted according to the 2013 Declaration of Helsinki guidelines.

\section{Immunohistochemistry}

The paraffin-embedded tissues were sliced into $4 \mu \mathrm{m}$ sections and baked at $75^{\circ} \mathrm{C}$ for 45 minutes. The sections were de-waxed in xylene and rehydrated in graded ethanol. Then, the slices were incubated in EDTA $(\mathrm{pH}=8.0)$ and $3 \% \mathrm{H}_{2} \mathrm{O}_{2}$ in methanol for 10 minutes. The tissue sections were treated with anti-NUCB2 antibodies (rabbit polyclonal antibody to NUCB2, ab224348; Abcam, Cambridge, UK) at 1:1,000 dilution and incubated overnight at $4^{\circ} \mathrm{C}$. Then the secondary antibody was added and incubated at room temperature for
30 minutes. After diaminobenzidine staining, the sections were counterstained using hematoxylin, dehydrated, and cleared with xylene. For the analysis of results, a semiquantitative H-score was computed for each sample by multiplying the staining intensities ( 0 : negative, 1 : weak staining, 2: moderate staining, 3: strong staining) and distribution areas $(0 \%-100 \%) .{ }^{17}$ All samples were classified into high-expression group and low-expression group according to the distribution of $\mathrm{H}$-score.

\section{Cell culture and transfection}

Both human thyroid cancer cell lines (TPC-1 and K1) used in this study were purchased from the Cell Bank of the Chinese Academy of Sciences (Shanghai, PR China). K1 was cultured in RPMI-1640 medium while TPC-1 was cultured in DMEM. Both contained 1\% penicillin streptomycin and $10 \%$ FBS. Cells were maintained at $37^{\circ} \mathrm{C}$ with $5 \% \mathrm{CO}_{2}$. For in vitro study, both TPC-1 and $\mathrm{K} 1$ cells were transfected with shRNA against NUCB2 to silence NUCB2 according to the manufacturer's instructions. The NUCB2 shRNA plasmids were transfected into thyroid cancer cells using Lipofectamine ${ }^{\circledR} 2000$ (Thermo Fisher Scientific, Waltham, MA, USA). The specific shRNA with the sequence of AAGCTGTGCCTATTGACATAGAC (Cat\# SH817843; Vigene Biosciences, Rockville, MD, USA) to target NUCB2 and scrambled sequence (negative control) were used. The cells were seeded at 50,000 cells/well in six-well plates according to the manufacturer's protocol, and three experimental groups were included: sh-NUCB2 group, which was transfected with shRNA targeting NUCB2; negative control group, which was transfected with scrambled sequence; and mock group, which was treated without transfection (data not shown). Silencing efficiency was measured by reverse-transcription PCR (RTPCR) and Western blot after 48 hours of transfection. These cells were used to explore the association between NUCB2 and cell proliferation and cell invasion. Then, the NUCB2 cell lines showing stable depletion were screened and used for the in vitro and in vivo assays.

To select the stable clones, transfected cells were selected and maintained in a medium containing $2 \mu \mathrm{g} / \mathrm{mL}$ puromycin. The stable cell lines were then verified by RT-qPCR and Western blot and used for later experiments.

\section{Reverse-transcription PCR}

The total RNA was extracted from tumor tissues and cells by using TRIzol ${ }^{\circledR}$ reagent (Thermo Fisher Scientific) according to the protocol. After measuring the content by 
ultraviolet analysis, RNA was reverse-transcribed to cDNA for qPCR analysis. Quantitative PCR was performed on a Smart Cycler using SGExcel FastSYBR Mixture (With Low ROX) Plus (Sango Biotech, Shanghai, PR China). To further analyze the real-time PCR data, we applied a comparative threshold cycle $(\mathrm{Ct})$ method which compares differences in $\mathrm{Ct}$ values between target RNA and common control. ${ }^{18}$ The sequences of primers are as follows: NUCB2: (forward) 5'-TCTTGGAGCCAGATAGCTGG-3' and (reverse) 5'-AGCTTCTGAGCCTCCAGTTG-3'; GAPDH: (forward) 5'-GAGTCAACGGATTTGGTCGT-3' and (reverse) 5'-TTGATTTTGGAGGGATCTCG-3'.

\section{Western blot}

Tissues and cells were lysed in RIPA lysis buffer, and protein concentrations were determined using the bicinchoninic acid method. Totally, $50 \mu \mathrm{g}$ of each protein was loaded per lane and separated on SDS-PAGE gels, and then transferred onto polyvinylidene fluoride membranes. After blocking with $5 \%$ dry milk for 1 hour at room temperature, primary monoclonal antibodies including NUCB2, mouse anti- $\beta$ actin (1:1,000 dilution, ab8226; Abcam), rabbit anti-Ki67 (1:1,000 dilution, ab16667; Abcam), mouse anti-PCNA (1:500 dilution, ab29; Abcam), mouse anti-MMP2 (1:1,000 dilution, ab37150; Abcam), and mouse anti-MMP9 (1:1,000 dilution, ab38898; Abcam) were used. The samples were then incubated with the secondary antibody (polyclonal goat antirabbit/mouse, 1:10,000 dilution; Rockland Immunochemicals Inc, Gilbertsville, PA, USA) for 30 minutes at $37^{\circ} \mathrm{C}$ and detected by chemiluminescence.

\section{Colony formation assay}

Both TPC-1 and K1 cells were seeded at a density of 500 cells/well and cultured on $60 \mathrm{~mm}^{2}$ dishes. Each group was measured in three parallel wells. After 10 days, cells were washed with PBS and then fixed with 10\% formaldehyde for 10 minutes. Cells were then stained with Giemsa stain for 10-20 minutes. The number of colonies was counted by using an optional microscope.

\section{MTT assay}

Both TPC-1 and K1 cells were seeded into 24-well dishes at a density of 3,000 cells/well, and $0.01 \mathrm{~mL}$ of $5 \mathrm{mg} / \mathrm{mL}$ MTT reagent was added into each well and incubated for 4 hours at $37^{\circ} \mathrm{C}$. After that, the medium was replaced with dimethyl sulfoxide (Sigma-Aldrich Co., St Louis, MO, USA) and kept for another 10 minutes. Microplate spectrophotometer
(Thermo Fisher Scientific) was employed to measure the OD at $570 \mathrm{~nm}$. All experiments were performed in triplicate.

\section{Transwell invasion assay}

Matrigel invasion chambers (Corning Incorporated, Corning, NY, USA) were used for the invasion assay. About $3 \times 10^{5}$ TPC-1 and K1 cells were isolated before adding to the upper chamber of transwell coated with Matrigel (BD Bioscience, Mountain View, CA, USA). After incubation for 24 hours at $37^{\circ} \mathrm{C}$ in $5 \% \mathrm{CO}_{2}$, the cells under the surface of the lower chamber were fixed with $4 \%$ paraformaldehyde solution and stained with $1 \%$ crystal violet. The numbers of invading cells were counted from five different randomly selected fields under a microscope (magnification, $\times 200$ ).

\section{Animal studies}

Five-week-old BALB/c nude mice (Slac Laboratory Animal Co. Ltd, Shanghai, PR China) were used for the tumorigenicity assay. About $1 \times 10^{7} \mathrm{~K} 1$ cells transfected with NUCB2 shRNA and controls were injected subcutaneously into the right rear flank of each mouse. Tumor volumes were measured using a caliper every week after 2 weeks by using the formula, tumor volume $=1 / 2 \times\left(\right.$ length $\times$ width $\left.^{2}\right)$. Seven weeks later, mice were sacrificed and tumors were harvested. All animal experiments in our research were approved by the Animal Experimentation Ethics Committee of Tianjin Medical University Cancer Institute and Hospital.

\section{Statistical analyses}

All the data in our study were analyzed by using SPSS 22.0. Data are presented as mean \pm standard error of the mean. Student's $t$-test was used for continuous variables, and chi-squared test was applied to analyze categorical variables. In this study, $P$-values $<0.05$ were considered to be statistically significant.

\section{Ethics approval and consent to participate}

All applicable international, national, and/or institutional guidelines for the care and use of human specimens and animals were followed. The animal study was carried out in accordance with the guidelines approved by the Animal Experimentation Ethics Committee of Tianjin Medical University Cancer Institute and Hospital. The protocol was approved by the committee, all surgeries were performed under sodium pentobarbital anesthesia, and all efforts were made to minimize the suffering. 


\section{Results}

\section{Correlation between NUCB2 and clinicopathological variables of PTC}

We investigated the correlation between NUCB2 protein expression status and clinicalpathological characteristics of patients via immunohistochemistry of tumor tissues from 155 patients with PTC. Both the nuclei and the cytoplasm of tumor cells were stained and showed typical strong and weak staining of NUCB2 (Figure 1A), while the NUCB2 expression was negative in the normal tissue adjacent to cancer (Figure 1B). The associations between NUCB2 protein expression and clinicopathological features are shown in Table 1. Besides, the results showed that NUCB2 was significantly associated with extrathyroidal extension, TNM stage, and tumor size $(P<0.05$, respectively, Table 1$)$. However, no associations were found between NUCB2 and age, gender, and lymph node metastasis ( $P>0.05$, respectively, Table 1$)$. Collectively, these results demonstrate the potential role of NUCB2 in PTC.

\section{Knocking down NUCB2 in TPC-I and $\mathrm{KI}$ cell lines}

To investigate the roles of NUCB2 in malignant biological behavior of PTC, we aimed to inhibit the expression of NUCB2 in thyroid cancer cells. NUCB2 expression was knocked down in both TPC-1 and $\mathrm{K} 1$ cell lines using NUCB2 shRNA. RT-qPCR and Western blot were performed to identify the expression of NUCB2 in shRNA and control groups. As shown in Figure 2A and B, the expression of NUCB2 was dramatically decreased in shRNA group compared to control group, which meant that we successfully knocked down NUCB2 expression in both cell lines.

\section{Downregulated expression of NUCB2 inhibited the proliferation of tumor cells}

Colony formation and MTT assays were performed to identify the role of NUCB2 in tumor cell proliferation. As indicated in Figure $3 \mathrm{~A}$ and $\mathrm{B}$, downregulation of the expression of NUCB2 significantly inhibited the proliferation of TPC-1 and K1 cells when compared to controls $(P<0.05)$. We then examined the expression status of some proliferation-related proteins including Ki67 and PCNA to explore the mechanism of action of NUCB2 in tumor cell proliferation. As expected, the expressions of Ki67 and PCNA were decreased when we silenced NUCB2, which was confirmed by Western blot (Figure $3 \mathrm{C}$ and $\mathrm{D}, P<0.05$ ). In summary, we found that downregulated expression of NUCB2 inhibited tumor cell proliferation via regulating the expression of proliferation-related proteins Ki67 and PCNA.

\section{Downregulated expression of NUCB2 inhibited tumor cell invasion}

Transwell assay was applied to explore whether NUCB2 also regulates migration and invasion, and we found that the invasion ability of shRNA group was dramatically decreased than that of the control group (Figure 4A,
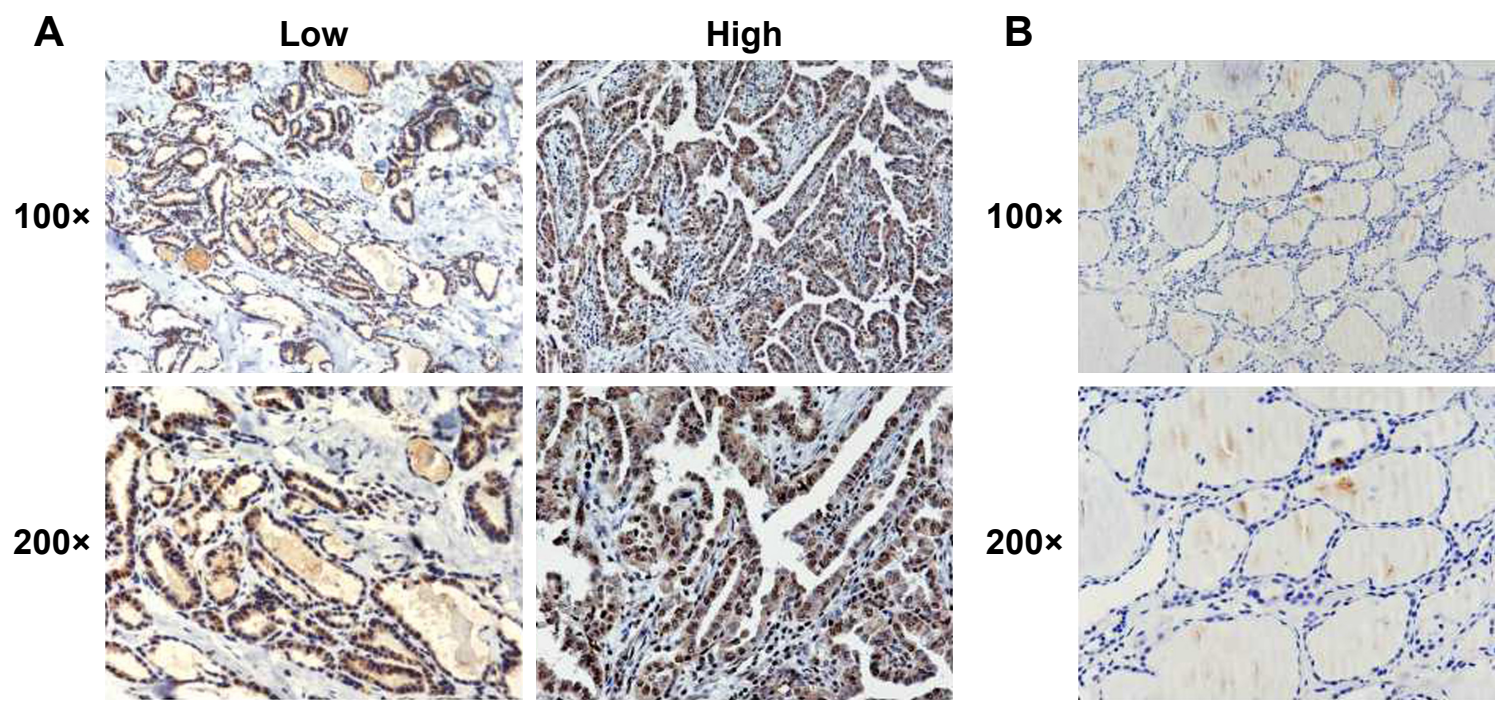

Figure I The expression of NUCB2 in human thyroid cancer and in the tissues adjacent to cancer.

Notes: (A) Represents immunohistochemical images of tissues showing high and low NUCB2 expression in human thyroid cancer at I00× and 200× optical magnifications. (B) Immunohistochemical images of NUCB2 expression in human tissues adjacent to cancer tissue at 100× and 200× optical magnifications. 
Table I Relationship of NUCB2 with clinicopathological characteristics in 155 patients with papillary thyroid cancer

\begin{tabular}{|c|c|c|c|c|c|}
\hline & \multirow[t]{2}{*}{$N=155$} & \multirow{2}{*}{\begin{tabular}{|l|} 
NUCB2 \\
Low \\
\end{tabular}} & \multirow{2}{*}{$\begin{array}{l}\text { expression } \\
\text { High }\end{array}$} & \multirow[t]{2}{*}{$\chi^{2}$} & \multirow[t]{2}{*}{ P-value } \\
\hline & & & & & \\
\hline Age (year) & & & & 0.025 & 0.874 \\
\hline$<55$ & 75 & 30 & 45 & & \\
\hline$\geq 55$ & 80 & 33 & 47 & & \\
\hline Gender & & & & 1.241 & 0.265 \\
\hline Male & 34 & II & 23 & & \\
\hline Female & 121 & 52 & 69 & & \\
\hline Extrathyroidal extension & & & & 6.500 & $0.011 *$ \\
\hline Yes & 113 & 39 & 74 & & \\
\hline No & 42 & 24 & 18 & & \\
\hline TNM stage & & & & 4.057 & $0.044 *$ \\
\hline $\mathrm{I}+\mathrm{II}$ & 135 & 59 & 76 & & \\
\hline $\mathrm{III+IV}$ & 20 & 4 & 16 & & \\
\hline Tumor size & & & & 6.575 & $0.010 *$ \\
\hline$<\mathrm{l} \mathrm{cm}$ & 67 & 35 & 32 & & \\
\hline$\geq 1 \mathrm{~cm}$ & 88 & 28 & 60 & & \\
\hline Lymph node metastasis & & & & 0.085 & $0.77 I$ \\
\hline Yes & 76 & 30 & 46 & & \\
\hline No & 79 & 33 & 46 & & \\
\hline Multifocality & & & & 1.008 & 0.315 \\
\hline Yes & 59 & 21 & 38 & & \\
\hline No & 96 & 42 & 54 & & \\
\hline
\end{tabular}

Note: $* P<0.05$.
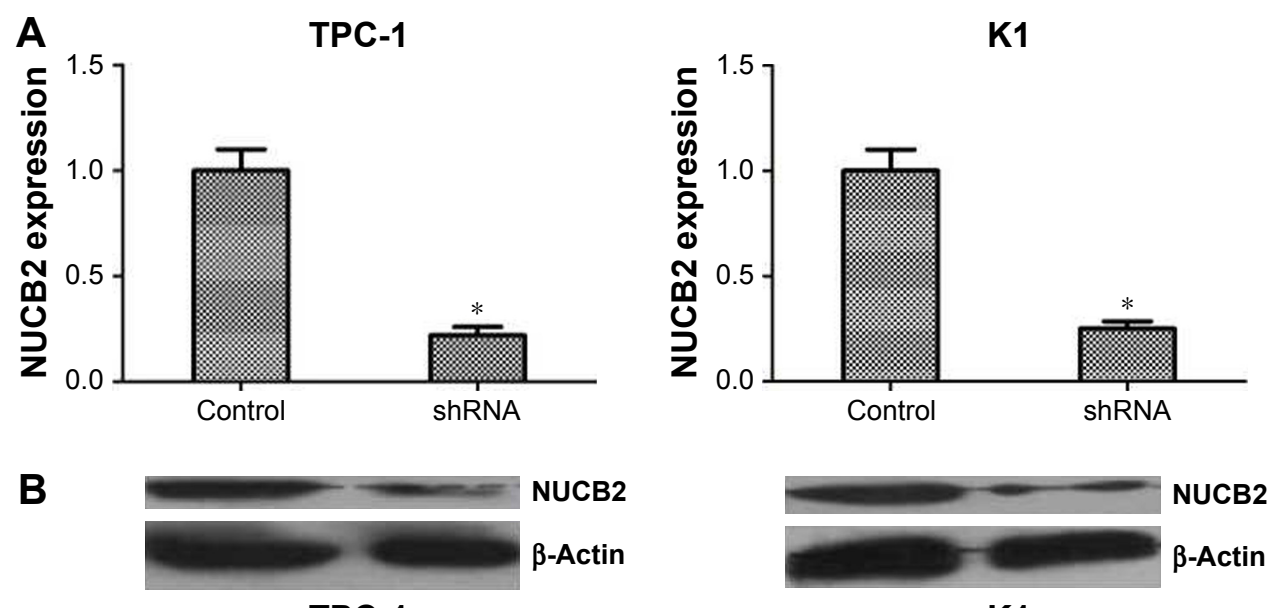

TPC-1
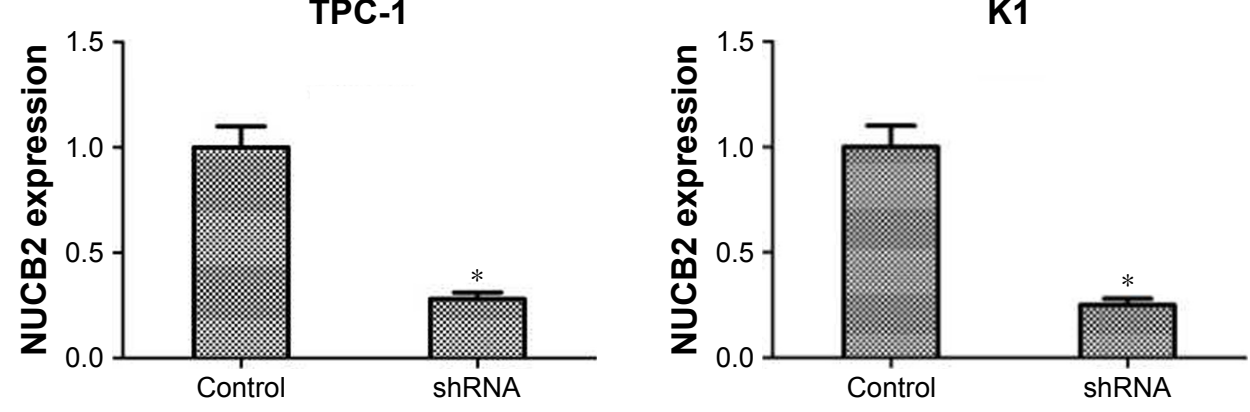

Figure 2 Knocking down of NUCB2 by shRNA in both TPC-I and KI cells.

Notes: RT-PCR (A) and Western blot (B) showed lower expression level of NUCB2 in shRNA group ( $n=3, P<0.05$, respectively), compared to the control group. $* P<0.05$. Abbreviation: RT-PCR, reverse-transcription PCR. 
A

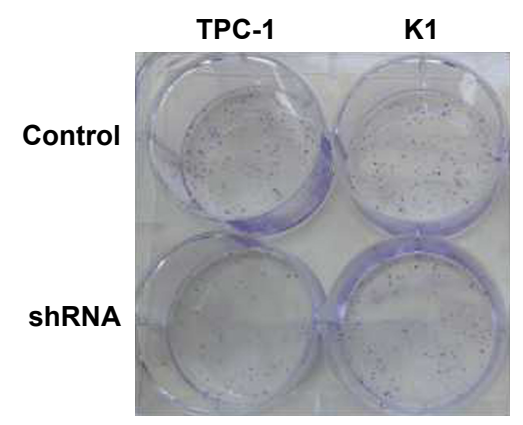

B

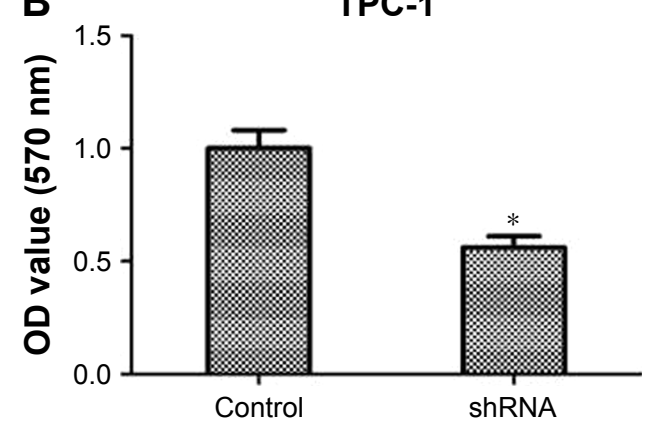

C

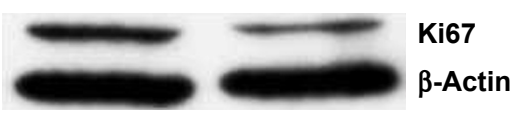

TPC-1

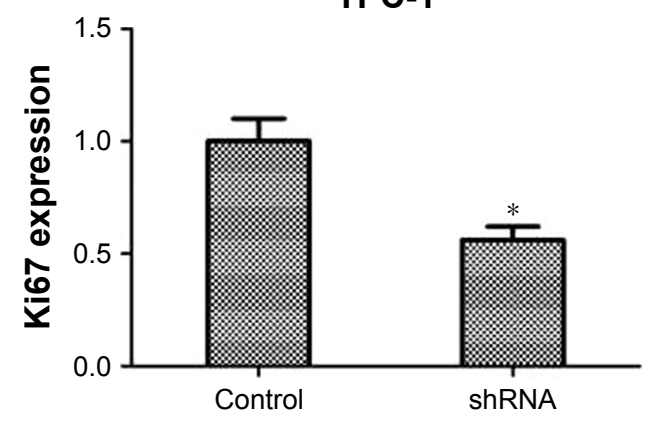

D

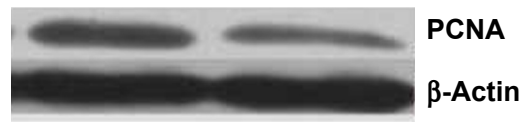

TPC-1

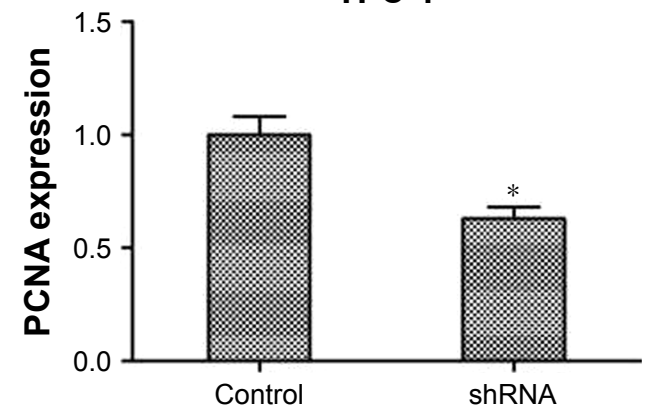

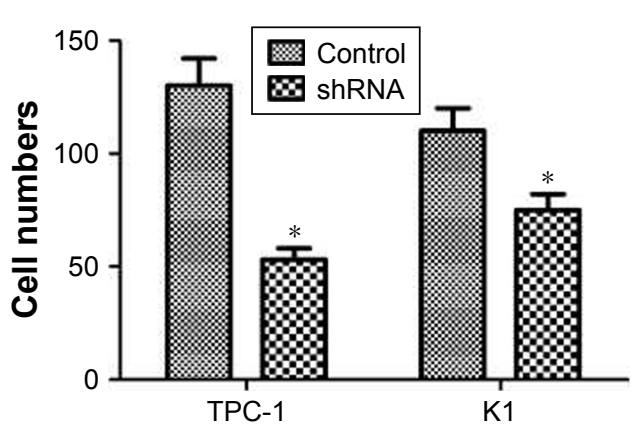

K1
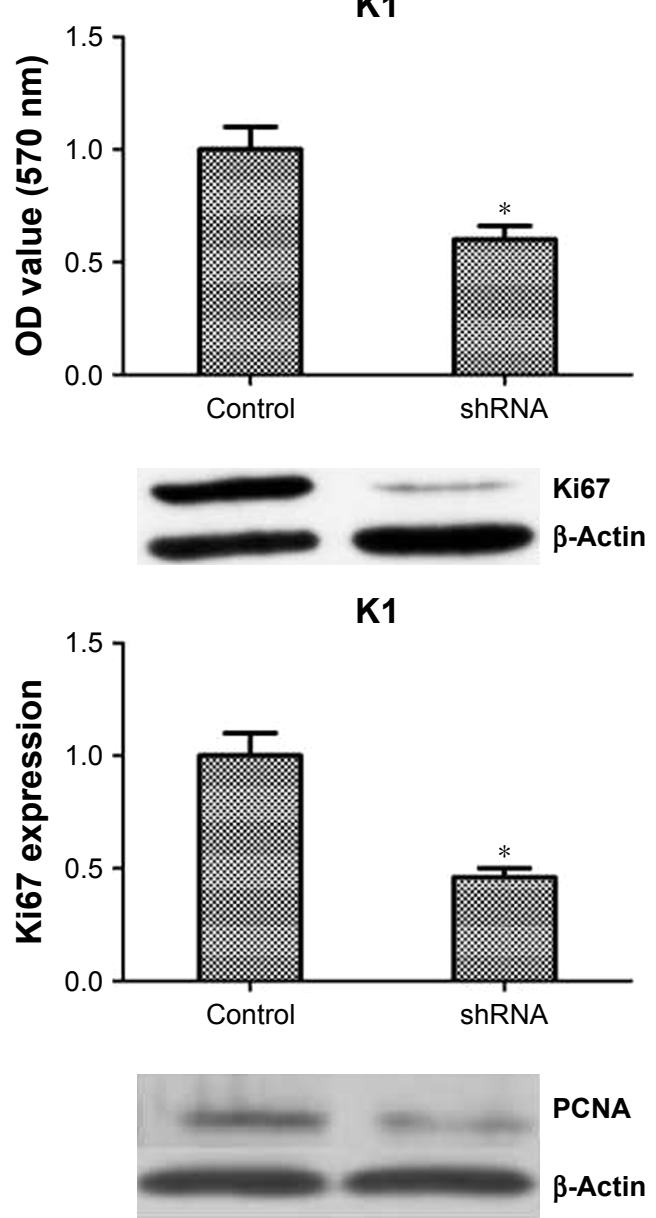

K1

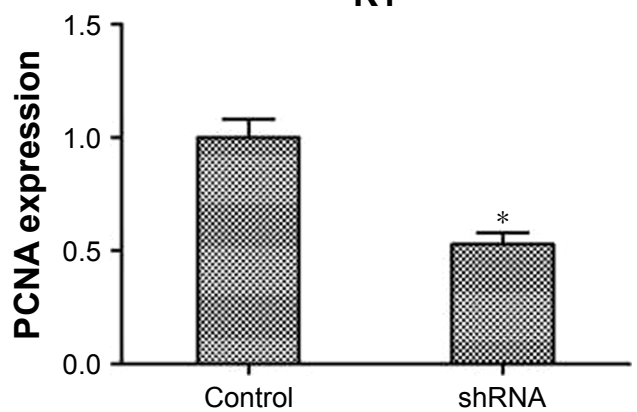

Figure 3 Knocking down NUCB2 in papillary thyroid cancer cells inhibited tumor cell proliferation by regulating Ki67 and PCNA.

Notes: (A) Typical images of colony-forming assay and its quantification demonstrated that colony rate of shRNA group was significantly lower than control group ( $n=3, P<0.05$, respectively). (B) OD value of MTT assay suggested that cell proliferation rate of shRNA group was lower than control group ( $n=3, P<0.05$, respectively). $(C$ and $\mathbf{D})$ Western blot and its quantification showed that the expression of Ki67 and PCNA was decreased in shRNA group when compared to control group ( $n=3, P<0.05$, respectively). $* P<0.05$. 
A

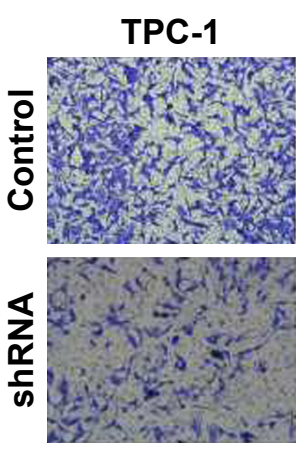

B

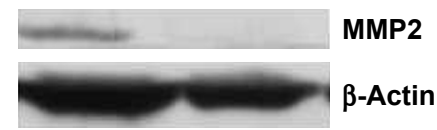

TPC -1

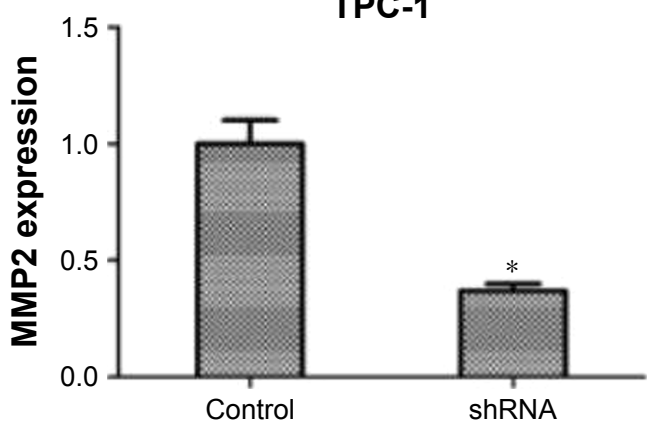

C

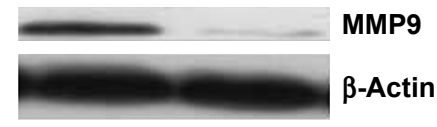

TPC-1

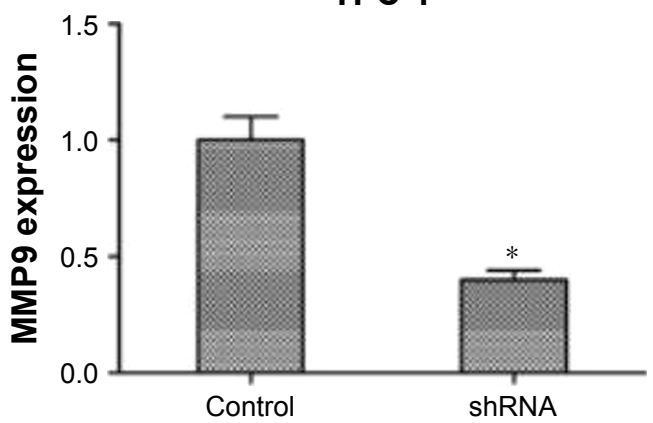

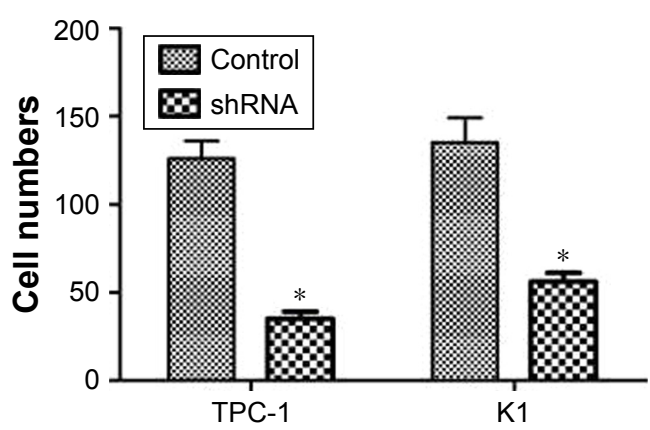

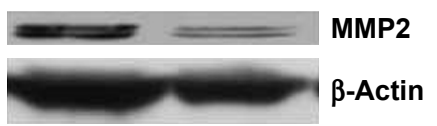

K1
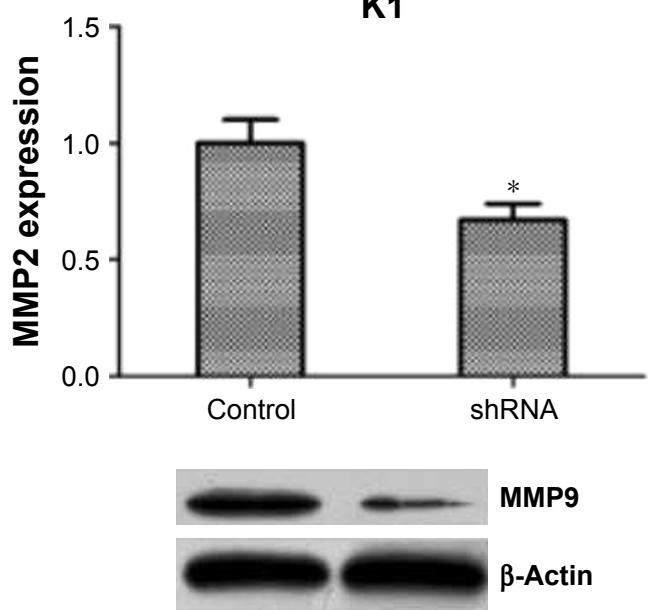

K1

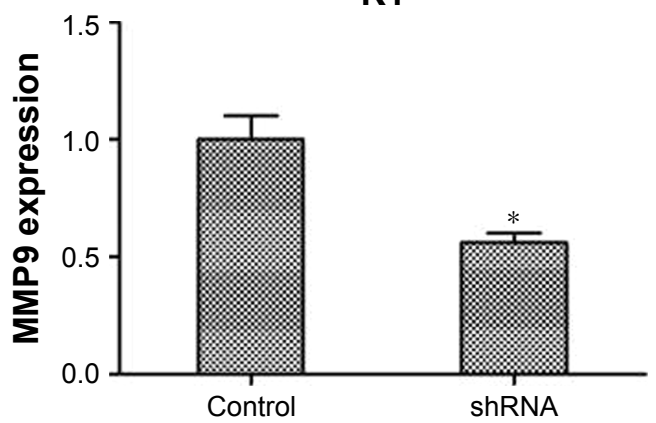

Figure 4 Downregulated expression of NUCB2 inhibited the migration and invasion of thyroid cancer cells by regulating MMP2 and MMP9.

Notes: (A) Transwell assay demonstrated that the ability of invasion in shRNA group was significantly decreased comparing to control group ( $\mathrm{n}=3, \mathrm{P}<0.05$, respectively). (B and $\mathbf{C}$ ). Western blot of MMP2 and MMP9 and its quantification showed that the expression of MMP2 and MMP9 were reduced in shRNA group when compared to control group $(n=3, P<0.05$, respectively). $* P<0.05$.

$P<0.05)$. To further explore the mechanism, we evaluated the expression of proteins related to tumor cell migration and invasion. By knocking down NUCB2, the expressions of MMP2 and MMP9 proteins were significantly decreased (Figure 4B and $\mathrm{C}, P<0.05$ ). In conclusion, we indicated that NUCB2 could promote the migration and invasion of thyroid tumor cells via modulating the expression of relevant proteins such as MMP2 and MMP9.

\section{Knocked down the expression of NUCB2 suppressed the tumorigenicity of PTC cells in vivo}

To further assess whether the in vitro behavior of NUCB2 could translate into abnormal tumorigenesis in vivo, cells from NUCB2 shRNA group and control group were injected subcutaneously into athymic mice. The tumor volumes were measured every week after 2 weeks. As shown in 

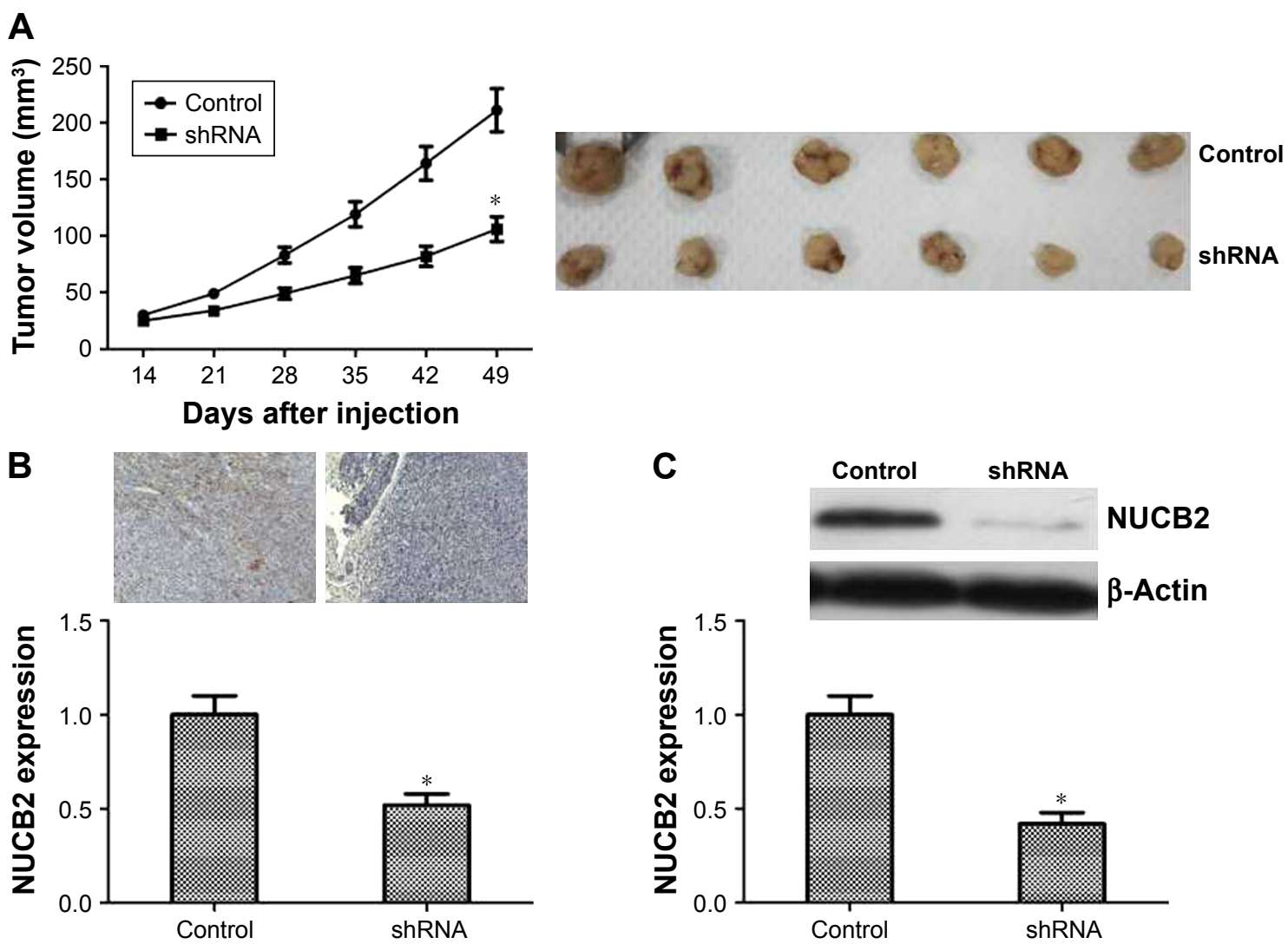

Figure 5 The influence of NUCB2 on tumor growth of thyroid cancer in mice.

Notes: $(\mathbf{A})$ Representative images of tumors from both groups are shown. Tumor volumes of shRNA group were smaller than those of the control group ( $n=6, P<0.05$ ). (B and C) Both immunohistochemistry and Western blot demonstrated that the expression of NUCB2 was dramatically decreased in mice tumors ( $n=6, P<0.05$, respectively), which suggested a successful construction of NUCB2 knocked down model in mice. $* P<0.05$.

Figure 5A, tumor growth in shRNA group was slower than that of the control group $(P<0.05)$. Besides, we also performed Western blot and immunohistochemistry analysis to detect the expression of NUCB2 in subcutaneous tumors. As shown in Figure 5B and C, NUCB2 expression was dramatically decreased in shRNA group, which indicated that an effective and stable knocking down of NUCB2 occurred in mice tumors.

\section{Discussion}

In this study, we investigated the expression of NUCB2 protein in human PTC tissues. Furthermore, we explored the function of NUCB2 in PTC aggression. PTC is well-differentiated and usually associated with good prognosis; nevertheless, about $10 \%$ of patients die from recurrences and distant metastasis after several years of diagnosis. ${ }^{4}$ A major clinical challenge is how to reliably distinguish patients who need aggressive treatments to reduce mortality from those who do not. ${ }^{19}$ Therefore, more studies on molecular modulation of thyroid cancer are needed to improve diagnosis and treatment in patients with clinically aggressive cancers.
NUCB2, an important protein broadly expressed in human hypothalamic nuclei and peripheral tissues, has drawn increasing attention because of its participation in multiple pathophysiological processes like nocturnal feeding and body weight regulation. ${ }^{12}$ However, a few recent studies have shown that there may be a correlation between NUCB2 and tumor behavior. Zeng et al found that NUCB2 protein was decreased in paired metastatic lymph node, with the positive expression rate being $82 \%$ in primary breast cancer tissue and $47 \%$ in paired metastatic lymph nodes. Furthermore, the overall survival time of patients with positive expression of NUCB2 protein was shorter compared to those with negative NUCB2 expression. ${ }^{20}$ These findings indicate that NUCB2 could be employed as a potential biomarker and a prognostic predictor for breast cancer. In addition, Zhang et al reported that high NUCB2 expression was positively related to seminal invasion, lymph node metastasis, and higher Gleason score in prostate cancer. They showed that patients with low NUCB2 protein expression had significantly better overall survival and biochemical recurrence-free survival compared with patients with high NUCB2 expression. ${ }^{21}$ 
Besides, NUCB2 was found to be related to tumor pathology and cancer-specific survival in renal cell carcinoma. ${ }^{17}$ In our study, the results suggested that high expression of NUCB2 was correlated with clinical pathological characters such as pTNM stage, extrathyroidal extension, and tumor size. All these results indicated that NUCB2 might be a potential prognostic marker and might play important roles in tumor progression.

Previous studies have revealed that NUCB2 is involved in tumor progression via regulation of cell proliferation and apoptosis in several cancers. ${ }^{13,14,22,23}$ In addition, Takagi et al reported that NUCB2 was associated with aggressiveness of endometrial carcinomas by promoting proliferation and migration of endometrial carcinoma cells. ${ }^{22}$ In vitro, downregulation of NUCB2 facilitated cell apoptosis and reduced the invasion rate in renal carcinoma. ${ }^{14,23}$ Similarly, in our research, knocking down of NUCB2 could significantly inhibit the proliferation and invasion of PTC cells in vitro. Moreover, the growth of tumors subcutaneously injected into mice was dramatically restricted by knocking down NUCB2. In conclusion, similar with other studies, our study indicated that NUCB2 functioned as a tumor promoter and could promote the progression of tumors both in vivo and in vitro.

There were still several limitations in our study. Foremost, to confirm whether NUCB2 could be used as a novel predictor of diagnosis, the finite sample size required another randomized study to investigate the correlation between NUCB2 expression and clinicopathological variables. In addition, our study only examined the proliferation and invasion of cells in which NUCB2 was downregulated. The relative specific signaling pathway requires more subsequent clarification.

\section{Conclusion}

Our study was the first to show the role of NUCB2 in tumor progression of PTC. As the results indicated, we speculated that NUCB2 was a tumor promoter in PTC, similar to its function in other carcinomas. However, more studies are required to verify and explore the exact mechanism underlying the function of NUCB2 in thyroid cancer in the future.

\section{Data sharing statement}

The dataset supporting the conclusion of this article is included within the article.

\section{Disclosure}

The authors report no conflicts of interest in this work.

\section{References}

1. Cramer JD, Fu P, Harth KC, Margevicius S, Wilhelm SM. Analysis of the rising incidence of thyroid cancer using the surveillance, epidemiology and end results National Cancer data registry. Surgery. 2010; 148(6):1147-1153.

2. Siegel RL, Miller KD, Jemal A, Oho A. Cancer statistics, 2018. CA Cancer J Clin. 2018;68(1):7-30.

3. DeSantis CE, Lin CC, Mariotto AB, et al. Cancer treatment and survivorship statistics, 2014. CA Cancer J Clin. 2014;64(4):252-271.

4. Xing M, Alzahrani AS, Carson KA, et al. Association between BRAF V600E mutation and mortality in patients with papillary thyroid cancer. JAMA. 2013;309(14):1493-1501.

5. Oh-IS, Shimizu H, Satoh T, et al. Identification of nesfatin- 1 as a satiety molecule in the hypothalamus. Nature. 2006;443(7112):709-712.

6. Miura K, Titani K, Kurosawa Y, Kanai Y. Molecular cloning of nucleobindin, a novel DNA-binding protein that contains both a signal peptide and a leucine zipper structure. Biochem Biophys Res Commun. 1992; 187(1):375-380.

7. Barnikol-Watanabe S, Gross NA, Götz H, et al. Human protein NEFA, a novel DNA binding/EF-hand/leucine zipper protein. Molecular cloning and sequence analysis of the cDNA, isolation and characterization of the protein. Biol Chem Hoppe Seyler. 1994;375(8):497-512.

8. Taniguchi N, Taniura H, Niinobe M, et al. The postmitotic growth suppressor necdin interacts with a calcium-binding protein (NEFA) in neuronal cytoplasm. J Biol Chem. 2000;275(41):31674-31681.

9. Islam A, Adamik B, Hawari FI, et al. Extracellular TNFR1 release requires the calcium-dependent formation of a nucleobindin 2-ARTS-1 complex. J Biol Chem. 2006;281(10):6860-6873.

10. Stengel A, Goebel M, Yakubov I, et al. Identification and characterization of nesfatin-1 immunoreactivity in endocrine cell types of the rat gastric oxyntic mucosa. Endocrinology. 2009;150(1):232-238.

11. Zhang AQ, Li XL, Jiang CY, et al. Expression of nesfatin-1/NUCB2 in rodent digestive system. WJG. 2010;16(14):1735-1741.

12. Cao X, Liu XM, Zhou LH. Recent progress in research on the distribution and function of NUCB2/nesfatin-1 in peripheral tissues. Endocr J. 2013;60(9):1021-1027.

13. Suzuki S, Takagi K, Miki Y, et al. Nucleobindin 2 in human breast carcinoma as a potent prognostic factor. Cancer Sci. 2012;103(1): 136-143.

14. Qi C, Ma H, Zhang HT, Gao JD, Xu Y. Nucleobindin 2 expression is an independent prognostic factor for clear cell renal cell carcinoma. Histopathology. 2015;66(5):650-657.

15. Zhang H, Qi C, Wang A, Li L, Xu Y. High expression of nucleobindin 2 mRNA: an independent prognostic factor for overall survival of patients with prostate cancer. Tumour Biol. 2014;35(3):2025-2028.

16. Qiu J, Zhang W, Xia Q, et al. Investigating the mechanisms of papillary thyroid carcinoma using transcriptome analysis. Mol Med Rep. 2017; 16(5):5954-5964.

17. Fu H, Zhu Y, Wang Y, et al. High NUCB2 expression level represents an independent negative prognostic factor in Chinese cohorts of non-metastatic clear cell renal cell carcinoma patients. Oncotarget. 2017;8(21): 35244-35254.

18. Livak KJ, Schmittgen TD. Analysis of relative gene expression data using real-time quantitative PCR and the 2(-Delta Delta C(T)) Method. Methods. 2001;25(4):402-408.

19. Lim H, Devesa SS, Sosa JA, Check D, Kitahara CM. Trends in thyroid cancer incidence and mortality in the United States, 1974-2013. JAMA. 2017;317(13):1338-1348.

20. Zeng L, Zhong J, He G, et al. Identification of Nucleobindin-2 as a potential biomarker for breast cancer metastasis using iTRAQbased quantitative proteomic analysis. J Cancer. 2017;8(15): 3062-3069.

21. Zhang H, Qi C, Wang A, et al. Prognostication of prostate cancer based on NUCB2 protein assessment: NUCB2 in prostate cancer. $J$ Exp Clin Cancer Res. 2013;32:77. 
22. Takagi K, Miki Y, Tanaka S, et al. Nucleobindin 2 (NUCB2) in human endometrial carcinoma: a potent prognostic factor associated with cell proliferation and migration. Endocr J. 2016;63(3):287-299.
23. Xu H, Li W, Qi K, Zhou J, Gu M, Wang Z. A novel function of NUCB2 in promoting the development and invasion of renal cell carcinoma. Oncol Lett. 2018;15(2):2425-2430.

\section{Publish your work in this journal}

OncoTargets and Therapy is an international, peer-reviewed, open access journal focusing on the pathological basis of all cancers, potential targets for therapy and treatment protocols employed to improve the management of cancer patients. The journal also focuses on the impact of management programs and new therapeutic agents and protocols on

patient perspectives such as quality of life, adherence and satisfaction. The manuscript management system is completely online and includes a very quick and fair peer-review system, which is all easy to use. Visit http://www.dovepress.com/testimonials.php to read real quotes from published authors. 